\title{
POLYCHLORINATED BIPHENYLS AS INDUCERS OF HEPATIC MICROSOMAL ENZYMES: STRUCTURE-ACTIVITY RULES
}

\author{
A. PARKINSON, L. ROBERTSON ${ }^{\text {, }}$, LORNA SAFE and S. SAFE* \\ Guelph-Waterloo Centre for Graduate Work in Chemistry, Department of Chemistry, \\ University of Guelph, Guelph, Ontario, N1G 2W1 (Canada) and ${ }^{a}$ School of Public \\ Health, The University of Michigan, Ann Arbor, MI 48109 (U.S.A.)
}

(Received December 28th, 1979)

(Revision received February 20th, 1980)

(Accepted February 25th, 1980)

\section{SUMMARY}

A number of highly purified polychlorinated biphenyl (PCB) isomers and congeners were synthesized and administered to male Wistar rats at dosage levels of 30 and $150 \mu \mathrm{mol} \cdot \mathrm{kg}^{-1}$. The effects of this in vivo treatment on the drug-metabolizing enzymes were determined by measuring the microsomal benzo[a]pyrene (B[a]P) hydroxylase, dimethylaminoantipyrine (DMAP) $N$-demethylase and NADPH-cytochrome $c$ reductase enzyme activities, the cytochrome $b_{5}$ content and the relative peak intensities and spectral shifts of the reduced microsomal cytochrome $P-450: C O$ and ethylisocyanide (EIC) binding difference spectra. The results were compared to the effects of administering phenobarbitone (PB), 3-methylcholanthrene (MC) and $\mathrm{PB}$ plus MC (coadministered) to the test animals. The synthetic PCB congeners used in this study included $3,4,4^{\prime}, 5$-tetrachlorobiphenyl (TCBP-1), 2,3',4,4' -tetrachlorobiphenyl (TCBP-2), 2,3',4,4',5'-pentachlorobiphenyl (PCBP-1), 2,3,4,$4^{\prime}, 5$-pentachlorobiphenyl (PCBP-2), 2,3, 3',4,4',5-hexachlorobiphenyl (HCBP1), 2,3,3', $4^{\prime}, 5,6$-hexachlorobiphenyl (HCBP-2), 2,3,3',5, $5^{\prime}, 6$-hexachlorobiphenyl (HCBP-3), 2,2',3,5,5',6-hexachlorobiphenyl (HCBP-4) and 2,3,3',4,$5,5^{\prime}$-hexachlorobiphenyl (HCBP-5) and were used to reappraise the structure-

*To whom reprint requests should be sent.

Abbreviations: $\mathrm{B}[a] \mathrm{P}$, benzo[a]pyrene; CO, carbon monoxide; DMAP, dimethylaminoantipyrene; EC, electron capture; EIC, ethylisocyanide; GLC, gas-liquid chromatography; HCBP-1, 2,3,3',4,4',5-hexachlorobiphenyl; HCBP-2, 2,3,3',4',5,6-hexachlorobiphenyl; HCBP-3, 2,3,3',5,5'-hexachlorobiphenyl; HCBP-4, 2,2',3,5, 5',6-hexachlorobiphenyl; HCBP-5, 2,3,3',4,5, $5^{\prime}$-hexachlorobiphenyl; MC, 3-methylcholanthrene; PB, phenobarbitone; PCB, polychlorinated biphenyl; PCBP-1, 2,3',4,4',5-pentachlorobiphenyl; PCBP2, 2,3,4,4',5-pentachlorobiphenyl, PMR, proton magnetic resonance, TCBP-1, 3,4,4',5tetrachlorobiphenyl; TCBP-2, 2,3 $, 4,4^{\prime}$-tetrachlorobiphenyl; TCDF, 2,3,7,8-tetrachlorodibenzofuran; TLC, thin layer chromatography. 
activity rules for PCBs as hepatic microsomal enzyme inducers. The results suggested that (a) PCBs which induce MC or mixed-type activity must be substituted at both para positions, at least two meta positions but not necessarily on the same phenyl ring and can also contain one ortho chloro substituent; (b) due to the considerable structural diversity of the PB-type inducers the rules for induction of this activity by PCB congeners are not readily defined.

\section{INTRODUCTION}

Commercial PCBs are complex mixtures of chlorinated biphenyl isomers and congeners $[1,2]$ which readily induce the hepatic drug-metabolizing enzymes. The spectral, enzymic and electrophoretic properties of hepatic microsomal monooxygenases induced by Aroclor 1254, a widely-used commercial PCB, are consistent with a pattern of mixed induction which can be simulated, for example, by coadministering the cytochrome $P-450$ inducer, $\mathrm{PB}$, with the cytochrome $P-448$ inducer, $\mathrm{MC}$ [3-9]. It has been proposed that the duality of the biochemical response to Aroclor 1254 and other commercial PCB mixtures is dependent on the presence of individual chlorinated biphenyls some of which induce cytochrome $P-450$ and some of which induce cytochrome $P-448$. In support of this proposal, several reports described the identification of individual PCB isomers and congeners as inducers of the microsomal monooxygenases [10-18] and their categorisation into PB-type or MC-type inducers [10-12,17,18]. From these studies on individual chlorinated biphenyls as microsomal monooxygenase inducers, the following structure-activity rules were suggested: (1) PCBs chlorinated in the ortho and para positions of both phenyl rings are PB-type inducers of cytochrome P-450-dependent monooxygenases; further chlorine substitution of PB-type inducers can alter the quantitative but not the qualitative effects of the inducer; (2) PCBs chlorinated in only the meta and para positions of both phenyl rings are MC-type inducers of cytochrome P-448-dependent monooxygenases; the addition of ortho chlorine substituents to MC-type inducers completely eliminates this activity; (3) PCBs chlorinated in only one phenyl ring and the lower chlorinated isomers and congeners are either weak or inactive as microsomal enzyme inducers.

A number of reports $[13,14]$ would appear to contradict the observations noted above. However, it has been pointed out that some of these inconsistencies may be due to 2,3,7,8-tetrachlorodibenzofuran, a potent inducer of cytochrome $P-448$, which may be present as a trace impurity in certain individual PCB preparations [19]. More recent studies with highly purified PCB isomers and congeners have shown that, in contrast to rule (2), certain compounds chlorinated in the meta and para positions of both rings and at only one ortho position are mixed (PB + MC)-type inducers [20,21]. Similarly, in contrast to rule (1), 2,2',3,3',5,5'-hexachlorobiphenyl, an isomer 
with no para chloro substituents, was shown to be a PB-type inducer [22,23].

It has been suggested that the toxicity of several classes of halogenated aromatic hydrocarbons is associated with those specific compounds which induce cytochrome P-448-dependent microsomal monooxygenases $[10,18]$. Therefore, the establishment of a structure-activity relationship for PCBs may assist in the identification of the more toxic PCB isomers and congeners. The relatively high toxicity of the possible MC-type inducers defined in rule (2) (namely $3,3^{\prime}, 4,4^{\prime}$-tetrachlorobiphenyl, 3,3',4,4',5-pentachlorobiphenyl and $3,3^{\prime}, 4,4^{\prime}, 5,5^{\prime}$-hexachlorobiphenyl) has been confirmed in previous studies $[15,16,24]$. In addition, some of the individual PCBs identified as mixed-type inducers also exhibited unusually high toxicity. For example $2,3,3^{\prime}, 4,4^{\prime}$-pentachlorobiphenyl and HCBP-1 were hepatotoxic to rats $[16,25]$ and the former also inhibited reproduction in chickens [26].

In view of the inconsistencies mentioned above and the predictive utility of the microsomal enzyme induction assay, a study was undertaken to further clarify the structural features that determine the activity of PCBs as microsomal enzyme inducers.

\section{MATERIALS AND METHODS}

Synthesis and purification of PCB isomers and congeners. 3,4-Dichloroaniline, 3,5-dichloroaniline, 2,5-dichloroaniline, 2,4-dichloroaniline, 4-chloroaniline, 1,2,3,4-tetrachlorobenzene, 1,2,4,5-tetrachlorobenzene and 1,2,3-trichlorobenzene were purchased from the Aldrich Chemical Co. and isoamyl nitrite was obtained from Eastman Organic Chemicals. The TCBP-1, PCBP-1, PCBP-2, HCBP-1, HCBP-2, HCBP-3, HCBP-4 and HCBP-5 were synthesized by the Cadogan coupling [27] of the appropriate chlorinated aniline (10-15 mmol) in excess of the chlorinated benzene $(200-300 \mathrm{mmol})$ in the presence of isoamyl nitrite $(20 \mathrm{mmol})$ as summarized in Table I. The reactions were carried out at $120^{-}-130^{\circ} \mathrm{C}$ for at least $18 \mathrm{~h}$ with rapid stirring. The excess chlorinated benzene was removed in vacuo and the crude reaction products were purified by column chromatography on alumina/Florisil and by thin-layer chromatography (TLC) as described [21]. The purified PCB congeners were crystallized from methanol and their structures confirmed by 220 or $60 \mathrm{MHz}$ proton magnetic resonance (PMR) spectroscopy (in deuterochloroform or deuteroacetone solvents respectively) and mass spectrometry. The spectroscopic data are summarized in Table I. The coupling reactions to give PCBP-2, HCBP-1, HCBP-2, HCBP-3, HCBP-4 and HCBP-5 gave only a single major PCB reaction product. TCBP-1 and PCBP-1 were each separated from one other major reaction product, namely 2,3,4,4'-tetrachlorobiphenyl and $2,2^{\prime}, 3,4,4^{\prime}$-pentachlorobiphenyl, respectively, by repeated TLC as previously reported [21]. 2,3',4,4'-Tetrachlorobiphenyl (TCBP-2) was synthesized as described [25]. The gas-liquid chromatographic (GLC) purities were determined using a Hewlett-Packard model 5710 chromatograph equipped with a ${ }^{63} \mathrm{Ni}$ electron capture (EC) or flame ionization 


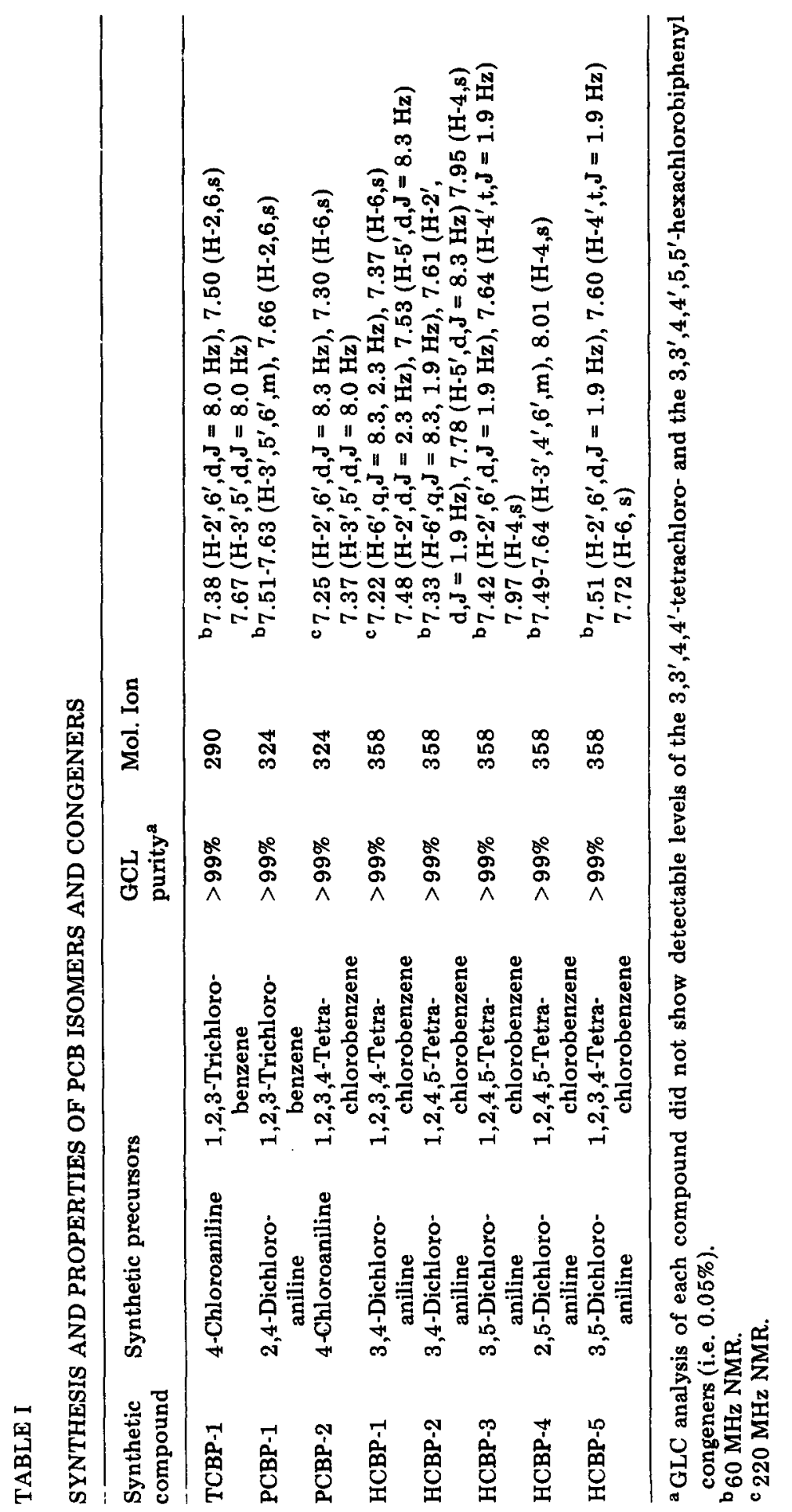


detector using an $0.6 \mathrm{~cm} \times 1.2 \mathrm{M}$ glass column packed with $3 \%$ OV 101 on Ultrabonded Carbowax 20M, 80-100 mesh (RFR Corp).

Biochemicals. Cytochrome $c$ (horse heart, type III), NADP ${ }^{+}, \mathrm{NADPH}$, $\alpha$-D-glucose-6-phosphate, $\alpha$-D-glucose-6-phosphate dehydrogenase (Baker's yeast), MC, B $[a] P$ and EIC were purchased from Sigma Chemical Co; DMAP from Aldrich Chemical Co; carbon monoxide (CO) (research purity) from Matheson and sodium phenobarbitone from the Ontario Veterinary College, Guelph. $\left[{ }^{3} \mathrm{H}\right] \mathrm{B}[a] \mathrm{P}$ was obtained from New England Nuclear Co. and purified by Florisil column chromatography using petroleum ether (30$\left.60^{\circ} \mathrm{C}\right)$ : methylene chloride $(99: 1, \mathrm{v} / \mathrm{v})$ as eluant.

Animal treatment and isolation of microsomes. One-month-old male Wistar rats, average weight $100 \mathrm{~g}$, were housed in wire cages and allowed free access to Purina Rat Chow and water. The PCB isomers and congeners were dissolved in corn oil and dosages of $30 \mu \mathrm{mol} \cdot \mathrm{kg}^{-1}$ (low dose) and $150 \mu \mathrm{mol} \cdot \mathrm{kg}^{-1}$ (high dose) were administered to four or five animals at each dose level by intraperitoneal injection on days 1 and 3 . The animals were killed by cervical dislocation on day 6 . PB $\left(400 \mu \mathrm{mol} \cdot \mathrm{kg}^{-1}\right)$ dissolved in isotonic saline and $\mathrm{MC}\left(100 \mu \mathrm{mol} \cdot \mathrm{kg}^{-1}\right)$ dissolved in corm oil were administered individually as well as coadministered to animals on days 1 and 2 and the animals killed on day 3 . Animals injected with a corresponding volume of vehicle $(0.5 \mathrm{ml}$ corn oil) served as controls which, along with PB-, MC- and/or PB + MC-treated rats, were included in each experiment and no significant variation was observed from week to week. All animals were fasted over the last $24 \mathrm{~h}$ to lower liver glycogen levels.

The rat livers were cleared of blood by perfusing via the hepatic portal vein with ice-cold isotonic saline supplemented with EDTA $(0.1 \mathrm{mM})$. The blanched livers were transferred to pre-weighed, ice-cold solutions of sucroseEDTA $(0.25 \mathrm{M}-0.1 \mathrm{mM})$ and the liver weights determined. The microsomal fraction was collected as a $100000 \times g$ pellet by further centrifugation of a $10000 \times g$ supernatant from the liver homogenate essentially as described [28].

Assays. In all assays the final concentration of microsomal protein was $1.0 \mathrm{mg} \cdot \mathrm{ml}^{-1}$ as determined by the method of Lowry et al. [29]. The cytochrome $P-450$ content was determined by the method of Omura and Sato [30], from the CO-difference spectrum of dithionite-reduced microsomes using an extinction coefficient of $91 \mathrm{~cm}^{-1} \cdot \mathrm{mM}^{-1}$ between $A_{\max }$ and $A_{490}$. The EIC-difference spectrum was determined in a similar manner to the CO-difference spectrum except that EIC was added to the sample cuvette (final concentration $4.5 \mathrm{mM}$ ) instead of CO. The absence of a peak at $420 \mathrm{~nm}$ in the difference spectrum between carbonmonoxyhaemoglobin and oxyhaemoglobin (determined by bubbling CO through the sample cuvette using oxidised microsomes) indicated that the microsomes used in this study were essentially free from haemoglobin contamination. The concentration of cytochrome $b_{5}$ was determined from the difference spectrum between NADH-reduced microsomes and oxidised microsomes [31] using the corrected extinction coefficient of $185 \mathrm{~cm}^{-1} \cdot \mathrm{mM}^{-1}$ [30]. All spectra were 
recorded on a Cary $118 \mathrm{C}$ spectrophotometer with a repetitive scan accessory. Holmium oxide was used to calibrate all spectra. In all cases, identical peak maxima from at least four of the five animals were recorded, hence, these results are expressed as the mode value.

All other assays were performed as described [28]. The rate of oxidative $\mathrm{N}$-demethylation of DMAP was measured by quantifying the production of formaldehyde. The formaldehyde, trapped as the semicarbazone, was developed in double-strength Nash reagent. The rate of $\mathrm{B}[a] \mathrm{P}$ hydroxylation was measured radiometrically by quantifying the base-soluble metabolites following hexane-extraction of the unreacted $\mathrm{B}[a] \mathrm{P}$. The activity of NADPH-cytochrome $P-450$ reductase was measured by the rate of the reduction of cytochrome $c$. To prolong the linearity of the cytochrome $c$ reduction, samples with high NADPH-cytochrome $P-450$ reductase activity were diluted to a final microsomal protein concentration of $0.2 \mathrm{mg} \cdot \mathrm{ml}^{-1}$.

The statistical significance between the sample means of control and treated groups for each parameter studied was analyzed by the Student's $t$-test at the $1 \%$ level of significance $(P<0.01)$.

\section{RESULTS}

The effects $P B, M C$ and their coadministration $(P B+M C)$. Induction of cyochromes $P-450$ by $P B$ was characterized by (a) a proliferation of the endoplasmic reticulum (as indicated by an increase in the $\mathrm{mg}$ microsomal protein $\mathrm{g}_{\text {liver }}{ }^{-1}$ ), (b) an increase in the activity of DMAP $N$-demethylase $(\times 2.3), \mathrm{B}[a] \mathrm{P}$ hydroxylase $(\times 3.0)$ and NADPH-cytochrome $c$ reductase ( $\times 2.3)$, (c) an increase in the concentration of cytochrome $b_{5}$ and $P-450$ with the absorption maximum of the latter occurring at $450.0 \mathrm{~nm}$ and (d) an EIC-difference spectrum displaying two peaks at 428.0 and $455.0 \mathrm{~nm}$ with a $455: 428 \mathrm{~nm}$ peak height ratio of about 0.7 . These features are shown in Table II.

In contrast, induction of cytochromes $P-448$ by $\mathrm{MC}$ was characterized by (a) no significant proliferation of the endoplasmic reticulum and no increase in the activity of DMAP $N$-demethylase or NADPH-cytochrome $c$ reductase, (b) a large increase in the activity of $\mathrm{B}[a] \mathrm{P}$ hydroxylase $(\times 14)$, (c) an increase in the concentration of cytochrome $b_{5}$ and $P-450$ with the absorption maximum of the latter shifted $2 \mathrm{~nm}$ downfield to $448.0 \mathrm{~nm}$ and (d) an EIC-difference spectrum with peak maxima at 429.6 and $452.0 \mathrm{~nm}$ and a $455: 428 \mathrm{~nm}$ peak height ratio of 1.9 .

In all cases the coadministration of $\mathrm{PB}$ and $\mathrm{MC}$ resulted in increases in enzymic activity and cytochrome concentration which were greater than the corresponding increases evoked by either $\mathrm{PB}$ or $\mathrm{MC}$ alone. Following the coadministration of $\mathrm{PB}$ and $\mathrm{MC}$, the $\mathrm{CO}$-difference spectrum showed maximum absorbance at $448.5 \mathrm{~nm}$, whilst the EIC-difference spectrum showed two peaks at 428.5 and $452.0 \mathrm{~nm}$ with a $455: 428 \mathrm{~nm}$ peak height ratio of 1.2. The spectral shifts elicited by the coadministration of $\mathrm{PB}$ and $\mathrm{MC}$ were slightly less than those evoked by the administration of $\mathrm{MC}$ alone. In general, 


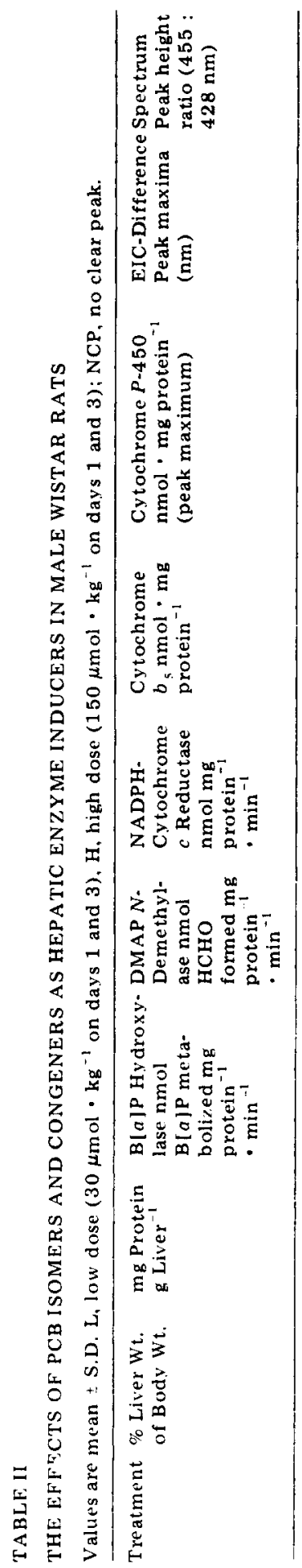

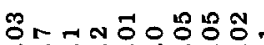

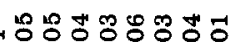
0000000000000000000000 $+1+1+1+1+1+1+1+1+1+1+1+1+1+1+1+1+1+1+1+1+1+1$

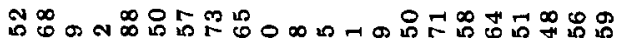
00 सं $00000000 \mathrm{0} 000000000000$

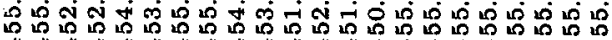

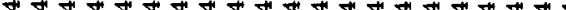

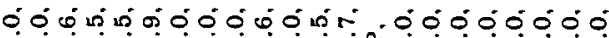

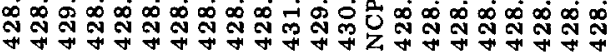

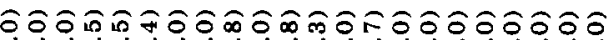

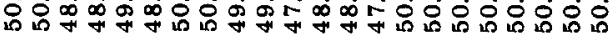

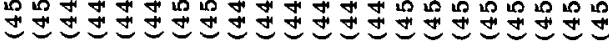
ஜึ? 0000000000000000000000 $+1+1+1+5+1+1+1+1+1+1+1+1+i+1+1+1+1+1+1+1+1+1$ ஜㄴำ

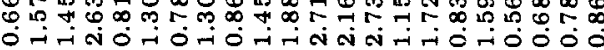

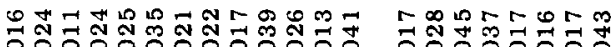

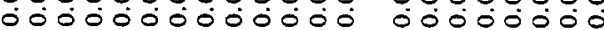

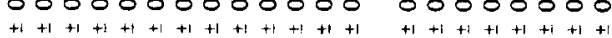

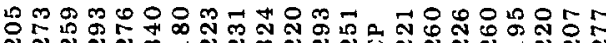

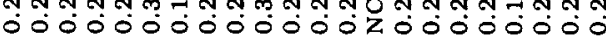

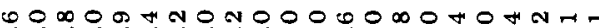

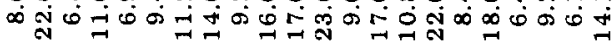

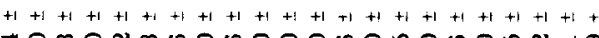

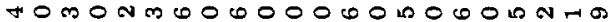

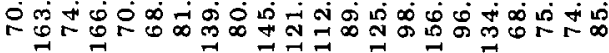

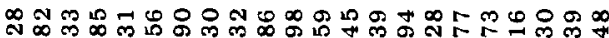

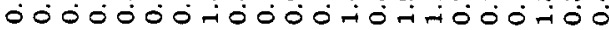
$+1+1+1+1+1+1+1+1+1+1+1+1+1+1+1+1+1+1+1+1+1+1$

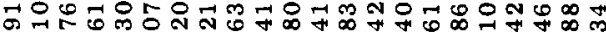

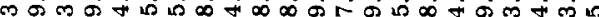

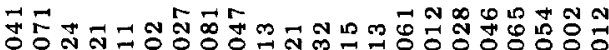
$00 \dot{0} 0 \dot{0} \dot{0} 0 \dot{0} 0000000000000 \dot{0}$ intitions

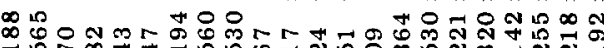
ón

0000 n 00 m no HON ก ล $+1+1+1+1+1+1+1+1+1+1+1+1+1+1+1$

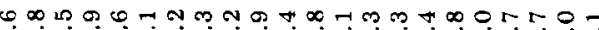

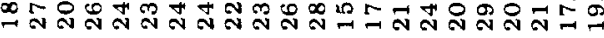

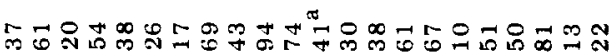

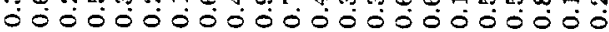
$+i+i+1+1+1+1+i+1+i+1+1+1+i+1+1+1+i+i+1+i+1+1$ ง 舟

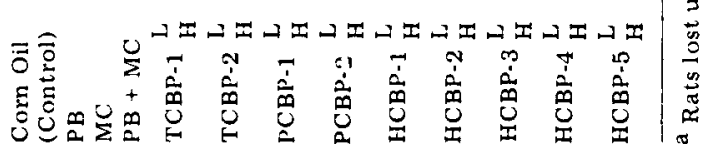


<smiles>Clc1ccc(-c2cccc(Cl)c2)cc1</smiles>

PCB 1<smiles>Clc1cccc(-c2ccccc2)c1</smiles>

PCB 2<smiles>Clc1ccc(-c2cc(Cl)c(Cl)c(Cl)c2)cc1</smiles>

TCBP 1<smiles>Clc1ccc(-c2ccc(Cl)c(Cl)c2)c(Cl)c1</smiles>

TCBP 2<smiles>Clc1ccc(-c2cc(Cl)c(Cl)c(Cl)c2)c(Cl)c1</smiles>

PCBP 1<smiles>Clc1ccc(-c2cc(Cl)c(Cl)c(Cl)c2Cl)cc1</smiles>

PCBP 2<smiles>Clc1ccc(-c2cc(Cl)c(Cl)c(Cl)c2Cl)cc1Cl</smiles>

HCBP 1<smiles>Clc1ccc(-c2c(Cl)c(Cl)cc(Cl)c2Cl)cc1Cl</smiles>

HCBP 2<smiles>Clc1cc(Cl)cc(-c2c(Cl)c(Cl)cc(Cl)c2Cl)c1</smiles>

HCBP 3<smiles>Clc1ccc(Cl)c(-c2c(Cl)c(Cl)cc(Cl)c2Cl)c1</smiles>

HCBP 4<smiles>Clc1cc(Cl)cc(-c2cc(Cl)c(Cl)c(Cl)c2Cl)c1</smiles>

HCBP 5

Fig. 1. The PCB isomers and congeners used in this study.

the qualitative spectral characteristics displayed by PB + MC- induced microsomes were intermediate between PB- and MC-induced microsomes.

It was shown elsewhere that the inducing effects of PB could be duplicated with $2,2^{\prime}, 4,4^{\prime}$-tetrachlorobiphenyl whilst $3,3^{\prime}, 4,4^{\prime}$-tetrachlorobiphenyl could simulate the inducing effects of MC [21]. This is an important observation since $2,2^{\prime}, 4,4^{\prime}$-tetrachlorobiphenyl and $3,3^{\prime}, 4,4^{\prime}$-tetrachlorobiphenyl are the simplest isomers satisfying structure-activity rules (1) and (2), respectively (Introduction). Therefore, for $\mathrm{PCBs}, 2,2^{\prime}, 4,4^{\prime}$-tetrachloro- 
biphenyl is a prototype PB-type inducer and $3,3^{\prime}, 4,4^{\prime}$-tetrachlorobiphenyl a prototype MC-type inducer.

The effects of selected PCB isomers and congeners. The results presented in Table II indicate that the PCB isomers and congeners tested were either PB-type, MC-type or mixed (PB + MC)-type inducers. The PB-type inducers included TCBP-2, HCBP-2 and HCBP-3. Pretreatment with these PCB isomers resulted in an increase in the concentration of cytochrome $b_{5}$ and $P-450$. Although increased in magnitude, the peak maxima of the COdifference spectrum $(450.0 \mathrm{~nm})$ and the EIC-difference spectrum $(428.0$ and $455.0 \mathrm{~nm})$ as well as the relative peak height ratio of the latter $(455: 428 \approx$ $0.5-0.7)$ were essentially unaltered compared to control values. The $2-$ to 3-fold increase in cytochrome $P-450$ concentration observed following pretreatment with TCBP-2, HCBP-2 and HCBP-3 at the high dose level $\left(150 \mu \mathrm{mol} \cdot \mathrm{kg}^{-1}\right)$ was accompanied by comparable increases in the activity of DMAP $N$-demethylase, $\mathrm{B}[a] \mathrm{P}$ hydroxylase and NADPH-cytochrome $c$ reductase. The effects of TCBP-2 confirmed earlier reports that this isomer is a PB-type inducer [12].

HCBP-2, -3 and -4 all share a common structural feature: namely, a 2,3,5,6-tetrachloro substitution pattern in one ring and one meta substituent in the other. Despite five of the six chlorine atoms being in identical positions, the position of the sixth chlorine greatly affected the potency with which these hexachlorobiphenyls induced cytochrome P-450. The sixth chlorine appears in the para, meta and ortho position in the lesser substituted ring of HCBP-2, HCBP-3 and HCBP-4, respectively. HCBP-2 was a more potent inducer of cytochrome $P-450$ than HCBP-3. This was particularly evident from their effects at the low dose level. However, HCBP-4 was either a non-inducer or a very weak PB-type inducer of cytochrome P-450.

TCBP-1 was found to be a MC-type inducer. In addition to an increase in its intensity, the Soret peak of the CO-difference spectrum was shifted $1.6 \mathrm{~nm}$ from $450 \mathrm{~nm}$ to $448.4 \mathrm{~nm}$ when TCBP-1 was administered at the high dose level. In the EIC-difference spectrum, both bathochromic and hypsochromic shifts were detected in the $428 \mathrm{~nm}$ and $455 \mathrm{~nm}$ peaks, respectively. Although neither DMAP $N$-demethylase nor NADPH-cytochrome $c$ reductase activity was increased, the activity of $\mathrm{B}[a] \mathrm{P}$ hydroxylase was enhanced 7.6- and 13-fold by TCBP-1 at the low and high dose, respectively. To date, only three PCB isomers $\left(3,3^{\prime}, 4,4^{\prime}\right.$-tetra, $3,3^{\prime}, 4,4^{\prime}, 5$-penta- and $3,3^{\prime}, 4,4^{\prime}, 5,5^{\prime}$-hexachlorobiphenyl) have been identified as strictly MC-type inducers. The results obtained for TCBP-1 indicated the existence of a fourth, pure MC-type inducer. It should be noted that, in accordance with rule (2) (Introduction), all pure MC-type inducers identified contain only meta and para substituents but, in contrast to the same rule, these positions need not be occupied in both phenyl rings as the results for TCBP-1 indicate.

It has been shown previously that, in contrast to rule (2), the addition of a single ortho substituent to each of the three PCBs identified as MC-type inducers (namely $3,3^{\prime}, 4,4^{\prime}$-tetra-, $3,3^{\prime}, 4,4^{\prime}, 5$-penta and $3,3^{\prime}, 4,4^{\prime}, 5,5^{\prime}$-hexachlorobiphenyl) did not abolish the MC-type effects with one exception 
TABLE III

PCB ISOMERS AND CONGENERS WHICH INDUCE HEPATIC CYTOCHROME $P-448$ DEPENDENT MONOOXYGENASES - IDENTIFICATION IN COMMERCIAL MIXTURES AND ENVIRONMENTAL SAMPLES

\begin{tabular}{|c|c|c|c|c|}
\hline \multirow[t]{2}{*}{ PCB Congener } & \multirow{2}{*}{$\begin{array}{l}\text { Type of } \\
\text { Inducer }\end{array}$} & \multicolumn{2}{|c|}{ Identification $^{a}$} & \multirow[t]{2}{*}{ Refs. } \\
\hline & & $\begin{array}{l}\text { Commercial } \\
\text { Mixtures }\end{array}$ & $\begin{array}{l}\text { Human } \\
\text { Tissue }\end{array}$ & \\
\hline $3,3^{\prime} ; 4,4^{\prime}$-Tetrachlorobiphenyl & MC & $\checkmark$ & ND & $1,2,34$ \\
\hline $3,4,4^{\prime}, 5$-Tetrachlorobiphenyl & MC & $?$ & ND & 1 \\
\hline $3,3^{\prime}, 4,4^{\prime}, 5$-Pentachlorobiphenyl & MC & ND & ND & 12 \\
\hline $2,3,4,4^{\prime}, 5$-Pentachlorobiphenyl & Mixed & ND & ND & This paper \\
\hline $2,3^{\prime}, 4,4^{\prime}, 5^{\prime}$-Pentachlorobiphenyl & Mixed & ND & ND & This paper \\
\hline $2,3,3^{\prime}, 4,4^{\prime}$-Pentachlorobiphenyl & Mixed & $\sqrt{ }$ & $\sqrt{ }$ & $1,2,40-42$ \\
\hline $2,3^{\prime}, 4,4^{\prime}, 5$-Pentachlorobiphenyl & Mixed & $y$ & $y$ & $1,2,40-42$ \\
\hline $2,3,3^{\prime}, 4,4^{\prime}, 5$-Hexachlorobiphenyl & Mixed & $\sqrt{ }$ & $\sqrt{ }$ & $1,2,40-42$ \\
\hline $3,3^{\prime}, 4,4^{\prime}, 5,5^{\prime}$-Hexachlorobiphenyl & MC & $\sqrt{ }$ & ND & 34 \\
\hline $2,3,3^{\prime}, 4,4^{\prime}, 5^{\prime}$-Hexachlorobiphenyl & Mixed & ND & ND & 21 \\
\hline $2,3,3^{\prime}, 4,4^{\prime}, 5,5^{\prime}$-Heptachlorobiphenyl & Mixed & $\sqrt{ }$ & $\sqrt{ }$ & 2,20 \\
\hline
\end{tabular}

${ }^{a}$ ND, not detected.

[21]. In addition to retaining their MC-type character, these PCBs also acquired PB-type properties and were classified, therefore, as mixed-type inducers. It was of interest to test whether TCBP-1, the newly identified pure MC-type inducer, would retain its MC-type character and acquire PB-type properties upon addition of a single ortho substituent. Substitution of TCBP-1 in one ortho position gives only two possible congeners, PCBP-1 and PCBP-2. Both PCBP-1 and PCBP-2 were found to be mixed-type inducers. That is, both pentachlorobiphenyls elicited MC-type effects, such as a large increase in $\mathrm{B}[a] \mathrm{P}$ hydroxylase activity, and PB-type effects, such as a 2 - to 3 -fold increase in DMAP $N$-demethylase and NADPH-cytochrome $c$ reductase activity. The quantitative and qualitative changes in both the COand EIC-difference spectra closely resembled those observed following the coadministration of PB and MC. Although both pentachlorobiphenyls were mixed-type inducers, PCBP-2 was found to be more potent than PCBP-1. It was shown previously that the mixed-type inducer, $2,3,3^{\prime}, 4,4^{\prime}$-pentachlorobiphenyl, was a more potent inducer of MC-type characteristics than PCBP-1 and that, whilst HCBP-1 was also a mixed-type inducer, $2,3^{\prime}, 4,4^{\prime}, 5,5^{\prime}$-hexachlorobiphenyl was an apparent PB-type inducer [21]. Based on these results and those obtained with PCBP-1 and PCBP-2, it appears that isomers with the single ortho substituent adjacent to a chloro substituent (i.e. the 2,3,4or 2,3,4,5-substitution pattern) are more potent inducers of MC-type characteristics than isomers with the single ortho substituent adjacent to a hydrogen (i.e. the 2,4- or 2,4,5- substitution pattern). 
Pretreatment with HCBP-5 at the high dose level resulted in increases in cytochrome $P-450(\times 1.3)$, DMAP $N$-demethylase $(\times 1.4)$ and NADPHcytochrome $c$ reductase $(X 1.2)$. These effects, together with the qualitative aspects of the spectral data, suggested that HCBP-5 is a weak PB-type inducer.

\section{DISCUSSION}

A complete assessment of the effect of structure on the activity of chlorinated biphenyls as microsomal monooxygenase enzyme inducers would require the synthesis and testing of all 209 possible congeners. This formidable task would also have to account for the in vivo formation of PCB metabolites, particularly from the lower chlorinated congeners (e.g. PCB-1 and PCB-2 in Fig. 1) and their possible role in the induction process. The PCBs used in this study were highly purified isomers and congeners containing a minimum of four chloro substituents (i.e. PCB-1; $x+y>4$ ). These more highly chlorinated compounds are not readily metabolized [32] and this obviates the need for identifying, synthesizing and ultimately testing their metabolites. In light of various exceptions to those structure-activity correlations described in Introduction, this study was designed to reappraise the proposed prerequisites (i.e. ortho, meta and para substitution patterns) for rat hepatic microsomal enzyme induction.

Structure-activity rules: PB-type inducers. It has been suggested that PB-type inducers require ortho and para chloro substituents on both phenyl rings (Introduction, rule (1)). The structural requirement for both para substituents was tested by evaluating the effects of a series of three hexachlorobiphenyl isomers, HCBP-1, HCBP-2 and HCBP-3, as microsomal enzyme inducers. We have previously reported that HCBP-1 (with two para substituents) was a mixed-type inducer and it is apparent from the results that pretreatment of rats with either HCBP-2 (with one para substituent) or HCBP-3 (with no para substituents) resulted in a PB-type enhancement of hepatic microsomal $\mathrm{B}[a] \mathrm{P}$ hydroxylase and DMAP $N$-demethylase activities and cytochrome $P-450$ content. A fourth isomer devoid of para substituents, HCBP-4, exhibited only marginal PB-type activity. These data and previous results reported for $2,2^{\prime}, 3,3^{\prime}, 5,5^{\prime}$-hexachlorobiphenyl [22,23] demonstrate that para chloro substitution of biphenyl is not a structural requirement for PB-type induction.

Previously we have shown that a number of PCB congeners substituted at only one ortho position (e.g. HCBP-1) were inducers of PB-type microsomal enzyme activities [20,21]. It has also been reported that administration of $4,4^{\prime}$-dichlorobiphenyl to rats resulted in proliferation of hepatic smooth endoplasmic reticulum and increases in hepatic microsomal aniline hydroxylase and $p$-nitroanisole- $O$-demethylase activities [33]. These data and others $[17,33]$ suggested that PCB congener(s) without any ortho chloro substituents can be PB-type inducers. It is possible that the activity observed for 
$4,4^{\prime}$-dichlorobiphenyl may be associated with one or more of its metabolites. However, in the rat the major metabolites of 4,4'-dichlorobiphenyl are 4,4'dichloro-3-biphenylol and $4^{\prime}$-chloro-4-biphenylol [32], both of which lack ortho substituents. Furthermore, 3,3'-dichlorobiphenyl is also a weak PBtype inducer $[11,17]$. Due to such variability it is apparent that the precise structural requirements for PCBs as PB-type inducers of microsomal enzyme activities cannot be readily defined.

Structure-activity rules: MC-type inducers. It was originally proposed that MC-type inducers require only para substitution and meta substitution on both phenyl rings (Introduction, rule (2)). However, the requirements for meta substitution have also not been unequivocally established. It has previously been shown that all mixed or MC-type inducers were substituted at one or two meta positions on both phenyl rings. TCBP-1, PCBP-1 and PCBP-2 also contain 2 meta-substituted chloro groups but these are now located on the same phenyl ring. The enzyme induction results clearly show that TCBP-1 is a pure MC-type inducer and PCBP-1 and PCBP-2 are both mixed-type inducers. It has previously been shown that TCBP-2 was a PBtype inducer [12]; this was confirmed in the present study and suggested that $\mathrm{PCB}$ isomers and congeners with MC-type activity must contain at least 2 meta chloro substituents, but not necessarily on the same ring.

Other reports $[10,12]$ and the data presented in this paper show that all mixed or MC-type inducers are substituted at both para positions and the loss of these substituents results in the loss of MC activity. HCBP-5 is an isomer of HCBP-1, a strong mixed-type inducer, in which one of the para (C-4') chloro substituents of the latter compound has been shifted to C-5' to give HCBP-5. This shift of a chloro substituent from a para to a meta position results in the complete loss of MC-type activity and supports the structural requirements of 2 para chloro substituents for this activity.

The recent identification of several mixed-type inducers $[20,21]$, e.g. HCBP-1, indicated that chloro substitution at one ortho position did not abolish the MC-type activity of these PCB congeners which were also substtuted in both para position and at least two meta positions. Presumably, the steric properties of only one ortho chloro substituent are not sufficient to hinder the formation of a coplanar PCB moiety which is isosteric with 2,3,7,8-tetrachlorodibenzo-p-dioxin and binds to the cytosol receptor protein involved in the initiation of microsomal monooxygenase enzyme induction [34]. The effects of 2 ortho chloro substituents might be expected to have a more pronounced effect on the population of the planar conformers and, hence, on their activity as MC-type inducers. Previous reports in the literature suggested that PCBs substituted in at least one meta and para position on each phenyl ring and at 2 ortho positions were strictly PB-type inducers as evidenced by the activity of the $2,2^{\prime}, 3,3^{\prime}, 4,4^{\prime}$ - and $2,2^{\prime}, 4,4^{\prime}, 5,5^{\prime}$ hexachlorobiphenyl isomers [10,11]. Contradictory reports have suggested that these isomers also possess MC-type activity [13,14]. A subsequent reinvestigation of the activity of $2,2^{\prime}, 4,4^{\prime}, 5,5^{\prime}$-hexachlorobiphenyl as a microsomal enzyme inducer has shown that the MC-type activity of a $99 \%$ 
pure commercial sample (prepared by the Ullman coupling method) was due to contamination with the highly active 2,3,7,8-tetrachlorodibenzofuran (TCDF) [19]. This result explains the apparent MC-type activity noted for this PCB isomer. Two papers have also reported that $2,2^{\prime}, 3,3^{\prime}, 4,4^{\prime}$-hexachlorobiphenyl and $2,2^{\prime}, 3,4,4^{\prime}, 5^{\prime}$-hexachlorobiphenyl were also mixed-type inducers after a 6-day [13] and a 90-day treatment regimen [14]. Since both isomers were synthesized by Ullman coupling procedures it is possible that these preparations may have also contained TCDF-like impurities; however, these apparent inconsistencies require further investigation.

By combining these data it is now possible to more accurately define the structure-activity rules for PCB isomers and congeners as hepatic microsomal monooxygenase enzyme inducers as follows: (1) PCBs which induce MC-type activity must be substituted at both para positions, at least two meta positions (but not necessarily on different phenyl rings) and can also contain one ortho substituent; (2) due to considerable diversity, the structure-activity rules for the induction of PB-type activity cannot be strictly defined.

Table III summarizes all the PCBs expected to exhibit MC-type activity based on the above guidelines and a number of these isomers and congeners have been identified in commercial PCBs $[1,2]$ and in humans (blood, adipose tissue and breast milk) [35-37]. Clearly, further testing is required to substantiate the correlation between the toxicity of these chemicals and their activities as inducers of cytochrome $P$-448-dependent monooxygenases. This may be significant in terms of human health since a comparison between the composition of the commercial PCB mixtures and the mixture of PCBs in humans revealed a preferential retention of the mixed-type inducers in human and animal tissues [ $35-37]$.

\section{ACKNOWLEDGEMENTS}

The financial assistance of the Research Programs Directorate Health and Welfare Canada, (606-1444-X), the National Cancer Institute (U.S.A.), HEW, Grant No. 1 BO1 CA21814-01, the Natural Sciences and Engineering Research Council of Canada and the United States Environmental Protection Agency (R806 928010 ) is gratefully acknowledged.

\section{REFERENCES}

1 D. Sissons and D. Welti, Structural identification of polychlorinated biphenyls in commercial mixtures by gas-liquid chromatography, nuclear magnetic resonance and mass spectrometry, J. Chromatogr., 60 (1971) 15.

$2 \mathrm{~S}$. Jensen and G. Sundström, Structures and levels of most chlorobiphenyls in two technical PCB products and in human adipose tissue, Ambio, 3 (1974) 70.

3 C.L. Litterst, T.M. Farber, A.M. Baker and E.J. Van Loon, Effect of polychlorinated biphenyls on hepatic microsomal enzymes in the rat, Toxicol. Appl. Pharmacol., 23 (1972) 112.

4 D.J. Ecobichon and A.M. Comeau, Comparative effects of commercial Aroclors on rat liver enzyme activities, Chem.-Biol. Interact., 9 (1974) 341. 
5 A.P. Alvares and A. Kappas, The inducing properties of polychlorinated biphenyls on hepatic monooxygenases, Clin. Pharmacol. Ther., 22 (1977) 809.

6 A.P. Alvares, D.R. Bickers and A. Kappas, Polychlorinated biphenyls : A new type of inducer of cytochrome $P-448$ in the liver, Proc. Natl. Acad. Sci. U.S.A., 70 (1973) 1321.

7 A.P. Alvares and A. Kappas, Heterogeneity of cytochrome $P \cdot 450$ s induced by polychlorinated biphenyls, J. Biol. Chem., 252 (1977) 6373.

8 D.E. Ryan, P.E. Thomas and W. Levin, Properties of purified liver microsomal cytochrome $P \cdot 450$ from rats treated with the polychlorinated biphenyl mixture Aroclor 1254, Mol. Pharmacol., 13 (1977) 521.

9 D.E. Ryan, P.E. Thomas, D. Korzeniowski and W. Levin, Separation and characterization of highly purified forms of liver microsomal cytochrome $P-450$ from rats treated with polychlorinated biphenyls, phenobarbital, and 3-methylcholanthrene, J. Biol. Chem., 254 (1979) 1365.

10 A. Poland and E. Glover, Chlorinated biphenyl induction of aryl hydrocarbon hydroxylase activity: a study of the structure-activity relationship, Mol. Pharmacol., 13 (1977) 924.

11 J.A. Goldstein, P. Hickman, H. Bergman, J.D. McKinney and M.P. Walker, Separation of pure polychlorinated biphenyl isomers into two types of inducers on the basis of induction of cytochrome $P-450$ or $P$-448, Chem.-Biol. Interact., 17 (1977) 69.

12 H. Yoshimura, N. Ozawa and S. Saeki, Inductive effects of polychlorinated biphenyls mixtures and individual isomers on the hepatic microsomal enzymes, Chem. Pharm. Bull., 26 (1978) 1215.

13 A.P. Alvares, Stimulatory effects of polychlorinated biphenyls (PCB) on cytochrome $P-450$ and $P-448$ mediated microsomal oxidations, in: V. Ullrich, (Ed.), Microsomes and Drug Oxidations, Pergamon Press, Inc. Oxford, 1977, p. 476.

14 M.D. Stonard and J.B. Greig, Different patterns of hepatic microsomal enzyme activity produced by administration of pure hexachlorobiphenyl isomers and hexachlorobenzene, Chem.-Biol. Interact., 15 (1976) 365.

15 H. Yoshimura, S. Yoshihara, N. Ozawa and M. Miki, Possible correlation between induction modes of hepatic enzymes by PCBs and their toxicity in rats, Ann. N.Y. Acad. Sci., 320 (1979) 179.

16 S. Yoshihara, K. Kawano, H. Yoshimura, H. Kuroki and Y. Masuda, Toxicological assessment of highly chlorinated biphenyl congeners retained in Yusho patients, Chemosphere, (1979) 531.

17 J.A. Goldstein, The structure activity relationships of halogenated biphenyls as enzyme inducers, Ann. N.Y. Acad. Sci., 320 (1979) 164.

18 A. Poland, W.F. Greenlee and A.S. Kende, Studies on the mechanism of action of the chlorinated dibenzo-p-dioxins and related compounds, Ann. N.Y. Acad. Sci., 320 (1979) 214.

19 J.A. Goldstein, J.R. Hass, P. Linko and D.J. Harvan, 2,3,7,8-Tetrachlorodibenzofuran in a commercially available $99 \%$ pure polychlorinated biphenyl isomer identified as the inducer of hepatic cytochrome $P$-448 and aryl hydrocarbon hydroxylase in the rat, Drug Metab. Disp., 6 (1978) 258.

20 A. Parkinson, R. Cockerline and S. Safe, Induction of both 3-methylcholanthrene and phenobarbitone-type activity by a single polychlorinated biphenyl isomer, Biochem. Pharmacol., 29 (1980) 259.

21 A. Parkinson, R. Cockerline and S. Safe, Polychlorinated biphenyl isomers and congeners as inducers of both 3-methylcholanthrene and phenobarbitone-type microsomal enzyme activity, Chem.-Biol. Interact., 29 (1980) 277.

22 K.K. Kohli, B.N. Gupta, P.W. Albro, H. Mukhtar and J.D. McKinney, Biochemical effects of hexachlorobiphenyl: fatty livers and cell structure, Chem.-Biol. Interact., 25 (1979) 139. 
23 K.K. Kohli, H. Mukhtar, J.R. Bend, P.W. Albro and J.D. McKinney, Biochemical effects of pure isomers of hexachlorobiphenyl-hepatic microsomal epoxide hydrase and cytosolic glutathione $S$-transferase activities in the rat, Biochem. Pharmacol., 28 (1979) 1444.

24 H.D. McKinney, K. Chae, B.N. Gupta, J.A. Moore and J.A. Goldstein, Toxicological assessment of hexachlorobiphenyl isomers and 2,3,7,8-tetrachlorodibenzofuran $I$. Relationship of chemical parameters, Toxicol. Appl. Pharmacol,, 36 (1976) 209.

25 H. Yamamoto, H. Yoshimura, M. Fujita and T. Yamamoto, Metabolic and toxicologic evaluation of $2,3,4,3^{\prime}, 4^{\prime}$-pentachlorobiphenyl in rats and mice, Chem. Pharm. Bull., 24 (1976) 2168 .

26 R.L. Ax and L.G. Hansen, Effects of purified PCB analogs on chicken reproduction, Poultry Sci., 54 (1975) 895.

27 J.I.G. Cadogan, A convenient new method of aromatic arylation, J. Chem. Soc., (Lond.), (1962) 4257.

28 A. Parkinson and S. Safe, The detection of enzyme induction by rat liver microsomes prepared by isoelectric precipitation, J. Pharm. Pharmacol., 31 (1979) 444.

29 O.H. Lowry, N.J. Rosebrough, A.L. Farr and R.J. Randall, Protein measurement with Folin phenol reagent, J. Biol. Chem., 193 (1951) 265.

30 T. Omura and R. Sato, The carbon monoxide-binding pigment of liver microsomes. I. Evidence for its haemoprotein nature, J. Biol. Chem., 239 (1964) 2370.

31 1. Raw and H.R. Mahler, Studies of electron transport enzymes. III. Cytochrome $b_{5}$ of pig liver mitochondria, J. Biol. Chem., 234 (1959) 1867.

32 G. Sundström, O. Hutzinger and S. Safe, The metabolism of chlorobiphenyls - a review, Chemosphere, 5 (1976) 267.

33 D.J. Ecobichon, M.M. Hansell and S. Safe, Halogen substituents at the 4 and $4^{\prime}$ positions of biphenyl: influence on hepatic function in the rat, Toxicol. Appl. Pharmacol., 42 (1977) 359.

34 A. Poland, E. Glover and A.S. Kende, Stereospecific, high affinity binding of 2,3,7,8tetrachlorodibenzo-p-dioxin by hepatic cytosol, J. Biol. Chem., 251 (1976) 4936.

35 T. Yakushiji, I. Watanabe, K. Kuwabara and S. Yoshida, Identification of low chlorinated biphenyls in human milk by gas chromatography-mass spectrometry, $J$. Chromatogr., 154 (1978) 203.

36 T. Yakushiji, I. Watanabe, K. Kuwabara, S. Yoshida, K. Koyama and N. Kunita, Levels of polychlorinated biphenyls (PCBs) and organochlorine residues in human milk and blood collected in Osaka prefecture from 1972-1977, Int. Arch. Occup. Environ. Health, 43 (1979) 1.

$37 \mathrm{H}$. Kuroki and $\mathrm{Y}$. Masuda, Structures and concentrations of the main components of polychlorinated biphenyls retained in patients with Yusho, Chemosphere, 6 (1977) 469. 